\title{
Parentage test in broad-snouted caimans (Caiman latirostris, Crocodylidae) using microsatellite DNA
}

\author{
Rodrigo B. Zucoloto ${ }^{1}$, Luciano M. Verdade ${ }^{2}$, Priscilla M.S. Villela ${ }^{3}$, Luciana C.A. Regitano ${ }^{4}$ \\ and Luiz L. Coutinho ${ }^{3}$ \\ ${ }^{1}$ Universidade Federal da Bahia, Salvador, BA, Brazil. \\ ${ }^{2}$ Laboratório de Ecologia Animal, Departamento de Ciências Biológicas, \\ Escola Superior de Agricultura Luiz de Queiroz, Universidade de São Paulo, Piracicaba, SP, Brazil. \\ ${ }^{3}$ Laboratório de Biotecnologia, Departamento de Zootecnia, Escola Superior de Agricultura Luiz de \\ Queiroz, Universidade de São Paulo, Piracicaba, SP, Brazil. \\ ${ }^{4}$ Embrapa Pecuária Sudeste, São Carlos, SP, Brazil.
}

\begin{abstract}
In this study, microsatellite markers, developed for Alligator mississipiensis and Caiman latirostris, were used to assess parentage among individuals from the captive colony of Caiman latirostris at the University of São Paulo, in Piracicaba, São Paulo, Brazil. Many of the females in the colony were full siblings, which made maternal identification difficult due to genotypic similarity. Even so, the most likely mother could be identified unambiguously among offspring in most of the clutches studied. Two non-parental females displayed maternal behavior which would have misled managers in assigning maternity based on behavior alone. This set of variable loci demonstrates the utility of parentage testing in captive propagation programs.
\end{abstract}

Key words: crocodilians, caiman, parentage, microsatellite DNA.

Received: October 16, 2008; Accepted: July 1, 2009.

Caiman latirostris is a medium-sized crocodilian that inhabits the wetlands and swamps of southeastern South America. The geographic distribution of the species covers the hydrographic basins of the Paraná and São Francisco Rivers, as well as a large number of small coastal drainage systems, from northeastern Brazil to northeastern Uruguay (Verdade, 1998; Verdade and Piña, 2006). The state of São Paulo, where this study was undertaken, is located in the center of the species range. Caiman latirostris was considered an endangered species in Brazil from 1972 to 2003 (Vanzolini, 1972; Groombridge, 1982; Bernardes et al., 1990; IBAMA 2003). The main causes for the decline in original populations were poaching for the leather trade, and habitat destruction, primarily for agricultural use (Brazaitis et al., 1988; Verdade, 1997).

Since the late 1980's, the Caiman latirostris conservation program developed by the University of São Paulo (ESALQ, Piracicaba, São Paulo, Brazil) has been successful in breeding this species in captivity (Verdade and Sarkis, 1998; Verdade et al., 2003). Due to the lack of informa-

Send correspondence to Rodrigo Barban Zucoloto. Laboratório de Genética de Populações e Evolução Molecular, Departamento de Biologia Geral, Instituto de Biologia, Universidade Federal da Bahia, Rua Barão de Geremoabo 147, Campus Universitário de Ondina, 40170-290 Salvador, BA, Brazil. E-mail: rbz@ufba.br. tion on nesting sites in the wild, and as this species is relatively common in Brazilian zoos, commercial farming operations have been proposed as the most feasible conservation strategy for the species in southern Brazil (Verdade, 1997, 2001).

Captive propagation efforts need to be guided by well-structured genetic management of the colony to prevent possible problems, such as founder effect, genetic drift and inbreeding depression (Ballou, 1992). Genetic management in the University of São Paulo captive colony is based on the establishment of a studbook in which individual pedigrees can be assessed and reproductive groups assembled, priority being given to nonrelated or leastrelated individuals (Verdade and Kassouf-Perina, 1993).

Molecular markers have been shown to be important tools in ecological and genetic research (Palo et al., 1995; Verdade et al., 2002). Microsatellites are among the best markers for parentage identification due to their high polymorphism (Craighead et al., 1995; Garcia-Moreno et al., 1996; Davis et al., 2001a), so that with enough markers, overall exclusion probabilities of $99.8 \%$ can be obtained.

Microsatellite markers specifically developed for $\mathrm{Al}$ ligator mississipiensis were tested with DNA from 21 species of the eight extant crocodilian genera (Glenn et al., 
1996, 1998). The tested primers were more efficient when amplifying orthologous loci in the DNA of species from the Alligatorinae subfamily than those from the Crocodylidae subfamily. However, amplification of Caiman latirostris DNA was not tested, and only one set of PCR conditions (the optimal conditions for American alligators) was used. Furthermore, the amount of intra-specific species polymorphism at the amplified loci was not determined for any of the other species. Therefore, it is possible that the use of different PCR conditions could permit amplification of additional loci from other species, especially Caiman latirostris. To date, there are 13 microsatellite markers specifically developed for Caiman latirostris (Zucoloto et al., 2002). Since only some microsatellite markers can be used among closely related species (Moore et al., 1991), we used microsatellite markers developed for Caiman latirostris and Alligator mississipiensis in the present study to assess parentage among individuals from the captive colony of Caiman latirostris at the University of São Paulo, in Piracicaba, São Paulo, Brazil.

The captive population sampled consisted of 16 adults and 24 hatchlings from the colony at the "Escola Superior de Agricultura - Luiz de Queiroz”, University of São Paulo, in Piracicaba, São Paulo, Brazil (Latitude: $22^{\circ}$ 42.556' S, Longitude: $47^{\circ} 38.246^{\prime} \mathrm{W}$ ). Individuals were identified in the pens by tail-notch marking and interdigital tags, but are represented here by their Regional Studbook number (Verdade and Kassouf-Perina, 1993; Verdade and Andrade, 2003). Samples studied and identified by CL are maintained in the lysis buffer collection of the "Laboratório de Biotecnologia", LPA, ESALQ, University of São Paulo, Piracicaba, São Paulo, Brazil. Alligatorinae. Caiman latirostris, Captive colony, ESALQ, University of São Paulo, Piracicaba, São Paulo, Brazil: 1-CL203, 2-CL25, 3-CL53, 4-CL106, 5-CL354, 6-CL355, 7-CL356, 8CL357, 121-CL458, 123-CL460, 124-CL461, 125-CL462, 33-CL30, 34-CL10, 35-CL5, 36-CL13, 37-CL14, 38CL70, 39-CL382, 40-CL383, 41-CL384, 42-CL385, 43CL386，63-CL434，64-CL435，65-CL436，67-CL438, 142-CL479, 144-CL481, 146-CL483, 82-CL1, 83-CL9, 84-CL2, 85-CL3, 86-CL4, 87-CL19, 88-CL406, 92CL410, 94-CL412, 96-CL414. Distribution of individuals in reproduction enclosures (ARN) was as follows: ARN1 (Father: 1-CL203, Possible mothers: 2-CL25, 3-CL53, 4CL106, Clutch 1: 5-CL354, 6-CL355, 7-CL356, 8-CL357, Clutch 5: 121-CL458, 123-CL460, 124-CL461, 125CL462); ARN3 (Father: 33-CL30, Possible mothers: 35-CL5, 34-CL10, 36-CL13, 37-CL14, 38-CL70, Clutch 2: 39-CL382, 40-CL383, 41-CL384, 42-CL385, 43-CL386, 8-CL357, Clutch 3: 63-CL434, 64-CL435, 65-CL436, 67-CL438, Clutch 6: 142-CL479, 144-CL481, 146-CL483) and ARN4 (Father: 82-CL1, Possible mothers: 84-CL2, 85-CL3, 86-CL4, 83-CL9, 87-CL19, Clutch 4: 88-CL406, 92-CL410, 94-CL412, 96-CL414). According to the Regional Studbook, the females 84-CL2, 85-CL3, 86-CL4,
35-CL5, 83-CL9, 34-CL10, 36-CL13, 37-CL14 and 87CL19 are full sisters.

Animal immobilization was mechanical without anesthetics or muscle relaxants (Verdade, 1997). Blood was collected from the dorsal branch of the superior cava vein, which runs along the interior of the vertebral column of large reptiles (Olson et al., 1975). After collection, blood was stored in a lysis buffer: $100 \mathrm{mM}$ Tris-HCl, $\mathrm{pH} 8.0$; $100 \mathrm{mM}$ EDTA, pH 8.0; $10 \mathrm{mM} \mathrm{NaCl} ; 0.5 \% \mathrm{SDS}$ (w/v) as in Hoelzel (1992). DNA from these samples was purified by CTAB and chloroform extraction followed by isopropyl alcohol precipitation (Sambrook et al., 1989).

Caimans build mound-nests, and females usually display parental behavior towards both the nest and hatchlings (Verdade, 1995; Thorbjarnarson, 1996). In this study, eggs were collected during the first $48 \mathrm{~h}$ after being laid and transferred to artificial incubators, (as described by Verdade et al., 1992). Eggs and resulting hatchlings were identified by nest. Females guarding the nest were identified and assigned as possible clutch-mothers.

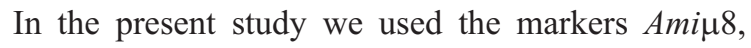

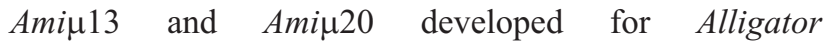
mississipiensis (Glenn et al., 1998) and the markers

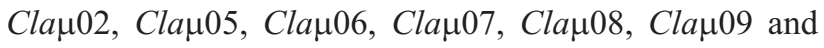
Cla 10 developed for C. latirostris (Zucoloto et al., 2002). Polymerase chain reaction (PCR) conditions were standardized for $25 \mu \mathrm{L}$ with: $1 \mathrm{X}$ specific buffer (Table 1, all buffers contain $300 \mathrm{mM}$ Tris- $\mathrm{HCl}$ and $75 \mathrm{mM}$ ammonium sulfate and differing concentrations of $\mathrm{Mg}^{2+}$ and $\mathrm{pH}$ ), $0.2 \mathrm{mM}$ each of $\mathrm{dNTP}, 0.4 \mu \mathrm{M}$ of each primer pair, $0.2 \mathrm{U}$ Taq DNA polymerase, and 100 ng DNA. The thermocycle program was: (1) $94{ }^{\circ} \mathrm{C}$ for $3 \mathrm{~min}$, (2) $94{ }^{\circ} \mathrm{C}$ for $1 \mathrm{~min}$, (3) primer specific annealing temperature for $1 \mathrm{~min}$, (4) $72{ }^{\circ} \mathrm{C}$ for $1 \mathrm{~min},(5)$ repeat steps 2,3 and 4 for $n$ cycles, (6) $72{ }^{\circ} \mathrm{C}$ for $7 \mathrm{~min}$ and $(7) 4{ }^{\circ} \mathrm{C}$ until storage (Table 1). Products were stored at $4{ }^{\circ} \mathrm{C}$ until analyzing and scoring. PCR products were loaded into a Megabace 1000 DNA sequencer system for genotyping. Primers were labeled according to Table 1 and individuals genotyped by using the Genetic profiler program.

For logical reasons, such as the movement of individuals being restricted to individual enclosures, statistics were estimated by considering enclosures as though they were sampling units, as described above, ARN1 $(\mathrm{N}=12)$ with one known parent (the father), three candidate parents (the possible mothers) and eight offspring from two clutches, ARN3 $(\mathrm{N}=18)$ with one known parent (the father), five candidate parents (the possible mothers) and twelve offspring from three clutches, ARN4 $(\mathrm{N}=10)$ with one known parent (the father), five candidate parents (the possible mothers) and four offspring from one clutch. The CERVUS 2.0 (Marshall et al., 1998) program was used for calculating exclusion power and null allele frequencies for each locus (Table 2). The overall probability of exclusion 
Table 1 - Primer and amplification conditions.

\begin{tabular}{|c|c|c|c|c|c|}
\hline Locus & Sequence $5^{\prime}-3$ ' & Buffer $10 X$ & Annealing ${ }^{\circ} \mathrm{C}$ & Cycles & Label \\
\hline Amin08a & CCTGGCCTAGATGTAACCTTC & $\mathrm{A}(7.5 \mathrm{mM} \mathrm{MgCl} 2, \mathrm{pH} 8.5)$ & 55 & 30 & FAM \\
\hline Ami $08 \mathrm{~b}$ & AGGAGGAGTGTGTTATTTCTG & & & & \\
\hline 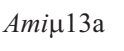 & CCATCCCCACCATGCCAAAGTC & $\mathrm{A}(7.5 \mathrm{mM} \mathrm{MgCl} 2, \mathrm{pH} 8.5)$ & 64 & 35 & FAM \\
\hline Ami $13 \mathrm{~b}$ & GTCCTGCTGCTGCCTGTCACT & & & & \\
\hline 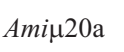 & TTTTTCTTCTTTCTCСАТТСТА & $\mathrm{F}(10 \mathrm{mM} \mathrm{MgCl} 2, \mathrm{pH} 9.0)$ & 58 & 30 & TET \\
\hline 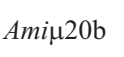 & GATCCAGGAAGCTTAAATACAT & & & & \\
\hline Cla $02 \mathrm{a}$ & CCTTCAGGACCCACTTTCTT & $\mathrm{A}(7.5 \mathrm{mM} \mathrm{MgCl} 2, \mathrm{pH} 8.5)$ & 58 & 30 & HEX \\
\hline Cla $\mu 2 \mathrm{~b}$ & CGAATCCСТCTTCCCAAACT & & & & \\
\hline Cla $05 \mathrm{a}$ & GCGTAGACAGATGCATGGAA & $\mathrm{F}(10 \mathrm{mM} \mathrm{MgCl} 2, \mathrm{pH} 9.0)$ & 55 & 30 & FAM \\
\hline Cla $05 \mathrm{~b}$ & CAGTCTGAAGCTAGGGCAAA & & & & \\
\hline Cla 06 a & GAAATATGGGACAGGGAGGA & $\mathrm{J}(10 \mathrm{mM} \mathrm{MgCl} 2, \mathrm{pH} 9.5)$ & 58 & 30 & TET \\
\hline Cla $\mu 66 \mathrm{~b}$ & GGTTGGCTGCATGTGTATGT & & & & \\
\hline Cla $07 \mathrm{a}$ & CGGGGTCTTGGTGTTGACTA & $\mathrm{F}(10 \mathrm{mM} \mathrm{MgCl} 2, \mathrm{pH} 9.0)$ & 58 & 30 & TET \\
\hline Cla $07 \mathrm{~b}$ & CGGGACCAGGAGCTGTATAA & & & & \\
\hline Cla $408 \mathrm{a}$ & CAGCCACTGAAGGAATTGAC & $\mathrm{F}(10 \mathrm{mM} \mathrm{MgCl} 2, \mathrm{pH} 9.0)$ & 55 & 30 & FAM \\
\hline Cla $08 \mathrm{~b}$ & CACATACCTGACCCAGCTTATC & & & & \\
\hline Cla $\mu 9^{\mathrm{a}}$ & ACAGGGGAAAAGAAGAGCTG & $\mathrm{A}(7.5 \mathrm{mM} \mathrm{MgCl} 2, \mathrm{pH} 8.5)$ & 60 & 35 & HEX \\
\hline Cla $09 \mathrm{~b}$ & AAAATCCCCCACTCTTACCC & & & & \\
\hline Cla $10 \mathrm{a}$ & TGGTCTTCTCTTCGTGTCCT & $\mathrm{A}(7.5 \mathrm{mM} \mathrm{MgCl} 2, \mathrm{pH} 8.5)$ & 60 & 35 & TET \\
\hline Cla $\mu 10 \mathrm{~b}$ & ATGAGCCCCTCTATGTTCCT & & & & \\
\hline
\end{tabular}

Table 2 - Descriptive statistics by enclosure.

\begin{tabular}{|c|c|c|c|c|c|c|c|c|c|c|c|c|}
\hline \multirow[t]{2}{*}{ Locus } & \multicolumn{4}{|c|}{ ARN1 } & \multicolumn{4}{|c|}{ ARN3 } & \multicolumn{4}{|c|}{ ARN4 } \\
\hline & $\mathrm{N}$ & $\operatorname{Excl}(1)$ & $\operatorname{Excl}(2)$ & Null & $\mathrm{N}$ & $\operatorname{Excl}(1)$ & $\operatorname{Excl}(2)$ & Null & $\mathrm{N}$ & $\operatorname{Excl}(1)$ & $\operatorname{Excl}(2)$ & Null \\
\hline Атін 08 & 12 & 0.099 & 0.173 & -0.200 & 18 & 0.060 & 0.143 & -0.124 & 10 & 0.000 & 0.000 & +0.000 \\
\hline 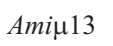 & 9 & 0.272 & 0.439 & +0.000 & 18 & 0.257 & 0.419 & -0.166 & 10 & 0.262 & 0.431 & -0.215 \\
\hline 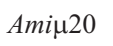 & 12 & 0.042 & 0.143 & -0.085 & 17 & 0.202 & 0.363 & -0.122 & 10 & 0.016 & 0.082 & -0.046 \\
\hline Cla 02 & 12 & 0.123 & 0.253 & -0.077 & 17 & 0.076 & 0.157 & +0.376 & 10 & 0.171 & 0.309 & -0.162 \\
\hline Cla 05 & 12 & 0.428 & 0.607 & -0.125 & 11 & 0.222 & 0.393 & -0.150 & 10 & 0.192 & 0.360 & +0.014 \\
\hline Clau06 & 12 & 0.217 & 0.382 & -0.044 & 9 & 0.194 & 0.340 & +0.000 & 7 & 0.146 & 0.258 & +0.000 \\
\hline Cla 07 & 12 & 0.162 & 0.304 & -0.145 & 17 & 0.189 & 0.329 & -0.113 & 10 & 0.125 & 0.188 & +0.111 \\
\hline Cla 008 & 12 & 0.391 & 0.569 & -0.132 & 18 & 0.069 & 0.194 & +0.033 & 9 & 0.309 & 0.481 & +0.000 \\
\hline Cla 09 & 12 & 0.215 & 0.363 & -0.181 & 18 & 0.070 & 0.152 & +0.385 & 6 & 0.162 & 0.304 & +0.000 \\
\hline \multirow[t]{2}{*}{ Cla 10} & 12 & 0.199 & 0.368 & -0.041 & 6 & 0.147 & 0.265 & +0.000 & 10 & 0.128 & 0.258 & -0.072 \\
\hline & & $0.921^{\mathrm{a}}$ & $0.991^{\mathrm{b}}$ & & & $0.806^{\mathrm{a}}$ & $0.964^{\mathrm{b}}$ & & & $0.816^{\mathrm{a}}$ & $0.963^{\mathrm{b}}$ & \\
\hline
\end{tabular}

N - Individuals analyzed; Excl(1) - Exclusion power with no known parent; Excl(2) - Exclusion power with one known parent known; Null - Null allele frequency estimates; ${ }^{\mathrm{a}}$ Total of exclusion power with no known parent; ${ }^{\mathrm{b}} \mathrm{Total}$ of exclusion power with one known parent.

for the maternity test by enclosure was computed with none parent known $(\operatorname{Excl}(1))$ or with one parent known $(\operatorname{Excl}(2))$ as shown in Table 2. CERVUS 2.0 was also used to assign maternity to possible mothers of offspring from the clutches in each enclosure, by employing the observed allele frequencies for enclosed populations to determine the statistical significance of the $\Delta$ value. This parameter was calculated by a simulation procedure that takes into account typing error rates and incomplete sampling for each possible mother, considering a given known father and offspring. At the end of this step, the possible mothers of each offspring were discriminated by $\Delta$ value and $\mathrm{CI}, e . g$. the confidence interval, which could be either $80 \%$ or $95 \%$, and corresponds to relaxed and restricted settings for CI, respectively, as shown in the last two columns of Table 3. 
Table 3 - Parentage test results by enclosure and clutch.

\begin{tabular}{|c|c|c|c|c|c|c|c|c|c|c|c|}
\hline & $\begin{array}{c}\text { Offspring } \\
\text { ID }^{\mathrm{a}}\end{array}$ & $\mathrm{KP} \mathrm{ID}^{\mathrm{b}}$ & KP class & $\begin{array}{l}\text { Offspring- } \\
\text { KP loci } \\
\text { compared }^{\mathrm{c}}\end{array}$ & $\begin{array}{c}\text { Prob. } \\
\text { non-exclus } \\
\text { ion }\end{array}$ & CP ID ${ }^{d}$ & $\begin{array}{l}\text { Offspring- } \\
\text { CP loci } \\
\text { compared }^{\mathrm{e}}\end{array}$ & $\begin{array}{l}\text { Offspring- } \\
\text { KP-CP loci } \\
\text { compared }^{f}\end{array}$ & LOD & Delta & CI \\
\hline \multirow[t]{4}{*}{ Clutch 1 (ARN1) } & $5(10)$ & $1(10)$ & Typed & $10(0)$ & $1.57 \mathrm{E}-03$ & $4(10)$ & $10(0)$ & $10(0)$ & $4.20 \mathrm{E}+00$ & $4.20 \mathrm{E}+00$ & $*$ \\
\hline & $6(10)$ & $1(10)$ & Typed & $10(0)$ & $3.14 \mathrm{E}-04$ & $4(10)$ & $10(0)$ & $10(0)$ & $6.38 \mathrm{E}+00$ & $6.38 \mathrm{E}+00$ & $*$ \\
\hline & $7(10)$ & $1(10)$ & Typed & $10(0)$ & $3.44 \mathrm{E}-03$ & $4(10)$ & $10(0)$ & $10(0)$ & $3.77 \mathrm{E}+00$ & $3.77 \mathrm{E}+00$ & $*$ \\
\hline & $8(10)$ & $1(10)$ & Typed & $10(0)$ & $2.92 \mathrm{E}-03$ & $4(10)$ & $10(0)$ & $10(0)$ & $4.14 \mathrm{E}+00$ & $4.14 \mathrm{E}+00$ & $*$ \\
\hline \multirow[t]{5}{*}{ Clutch 2 (ARN3) } & $39(7)$ & $33(9)$ & Untyped & $6(0)$ & $2.37 \mathrm{E}-01$ & $35(10)$ & $7(0)$ & $6(0)$ & $1.93 \mathrm{E}+00$ & $5.86 \mathrm{E}-01$ & + \\
\hline & $40(7)$ & $33(9)$ & Untyped & $6(0)$ & $2.19 \mathrm{E}-01$ & $35(10)$ & $7(0)$ & $6(0)$ & $1.90 \mathrm{E}+00$ & $6.79 \mathrm{E}-01$ & + \\
\hline & $41(8)$ & $33(9)$ & Untyped & $7(0)$ & $8.11 \mathrm{E}-02$ & $34(10)$ & $8(0)$ & $7(0)$ & $2.76 \mathrm{E}+00$ & $1.30 \mathrm{E}+00$ & + \\
\hline & $42(7)$ & $33(9)$ & Untyped & $6(0)$ & $5.52 \mathrm{E}-02$ & $34(10)$ & $7(0)$ & $6(0)$ & $3.08 \mathrm{E}+00$ & $6.53 \mathrm{E}-01$ & + \\
\hline & $43(8)$ & $33(9)$ & Untyped & $7(0)$ & $8.16 \mathrm{E}-02$ & $34(10)$ & $8(0)$ & $7(0)$ & $2.93 \mathrm{E}+00$ & $1.23 \mathrm{E}+00$ & + \\
\hline \multirow[t]{4}{*}{ Clutch 3 (ARN3) } & $63(8)$ & $33(9)$ & Untyped & $7(0)$ & $1.81 \mathrm{E}-01$ & $35(10)$ & $8(0)$ & $7(0)$ & $2.57 \mathrm{E}+00$ & $6.79 \mathrm{E}-01$ & + \\
\hline & $64(7)$ & $33(9)$ & Untyped & $6(0)$ & $2.05 \mathrm{E}-01$ & $35(10)$ & $7(0)$ & $6(0)$ & $2.16 \mathrm{E}+00$ & $6.79 \mathrm{E}-01$ & + \\
\hline & $65(7)$ & $33(9)$ & Untyped & $6(0)$ & $2.28 \mathrm{E}-01$ & $35(10)$ & $7(0)$ & $6(0)$ & $2.08 \mathrm{E}+00$ & $6.79 \mathrm{E}-01$ & + \\
\hline & $67(8)$ & $33(9)$ & Untyped & $7(0)$ & $3.63 \mathrm{E}-02$ & $35(10)$ & $8(0)$ & $7(0)$ & $4.21 \mathrm{E}+00$ & $3.49 \mathrm{E}+00$ & $*$ \\
\hline \multirow[t]{4}{*}{ Clutch 4 (ARN4) } & $88(9)$ & $82(10)$ & Typed & $9(0)$ & $1.38 \mathrm{E}-01$ & $87(10)$ & $9(0)$ & $9(0)$ & $2.72 \mathrm{E}+00$ & $1.06 \mathrm{E}+00$ & $*$ \\
\hline & $92(8)$ & $82(10)$ & Typed & $8(0)$ & $1.69 \mathrm{E}-01$ & $87(10)$ & $8(0)$ & $8(0)$ & $2.28 \mathrm{E}+00$ & $5.49 \mathrm{E}-01$ & + \\
\hline & $94(7)$ & $82(10)$ & Typed & $7(0)$ & 3.04E-01 & $87(10)$ & $7(0)$ & $7(0)$ & $2.07 \mathrm{E}+00$ & $6.51 \mathrm{E}-01$ & + \\
\hline & $96(8)$ & $82(10)$ & Typed & $8(0)$ & $1.47 \mathrm{E}-01$ & $87(10)$ & $8(0)$ & $8(0)$ & $2.37 \mathrm{E}+00$ & $5.49 \mathrm{E}-01$ & + \\
\hline \multirow[t]{4}{*}{ Clutch 5 (ARN1) } & $121(10)$ & $1(10)$ & Typed & $10(0)$ & $1.65 \mathrm{E}-03$ & $3(10)$ & $10(0)$ & $10(0)$ & $6.23 \mathrm{E}+00$ & $6.23 \mathrm{E}+00$ & $*$ \\
\hline & $123(10)$ & $1(10)$ & Typed & $9(0)$ & $8.27 \mathrm{E}-03$ & $3(10)$ & $9(0)$ & $9(0)$ & $3.96 \mathrm{E}+00$ & $3.96 \mathrm{E}+00$ & $*$ \\
\hline & $124(10)$ & $1(10)$ & Typed & $9(0)$ & $5.72 \mathrm{E}-03$ & $3(10)$ & $9(0)$ & $9(0)$ & $4.70 \mathrm{E}+00$ & $4.70 \mathrm{E}+00$ & $*$ \\
\hline & $125(10)$ & $1(10)$ & Typed & $9(0)$ & $1.71 \mathrm{E}-02$ & $3(10)$ & $9(0)$ & $9(0)$ & $3.34 \mathrm{E}+00$ & $3.34 \mathrm{E}+00$ & $*$ \\
\hline \multirow[t]{3}{*}{ Clutch 6 (ARN3) } & $142(8)$ & $33(9)$ & Untyped & $7(0)$ & $1.87 \mathrm{E}-01$ & $35(10)$ & $8(0)$ & $7(0)$ & $2.29 \mathrm{E}+00$ & $6.91 \mathrm{E}-01$ & + \\
\hline & $144(7)$ & $33(9)$ & Untyped & $6(0)$ & $2.19 \mathrm{E}-01$ & $36(10)$ & $7(0)$ & $6(0)$ & $5.92 \mathrm{E}-01$ & $5.92 \mathrm{E}-01$ & + \\
\hline & $146(8)$ & $33(9)$ & Untyped & $7(0)$ & $3.29 \mathrm{E}-02$ & $36(10)$ & $8(0)$ & $7(0)$ & $2.58 \mathrm{E}+00$ & $2.09 \mathrm{E}+00$ & $*$ \\
\hline
\end{tabular}

IDs in this table correspond to laboratory number. In the confidence interval column (CI) a + signal indicates that the result lies in the $80 \%$ confidence interval and an ${ }^{*}$ signal indicates that the result lies on the $95 \%$ confidence interval.; ${ }^{\mathrm{a}}$ (Offispring loci typed); ${ }^{\mathrm{b}}\left(\mathrm{Known}\right.$ Parent loci typed); ${ }^{\mathrm{c}}(\mathrm{Off}-$ spring-Known Parent loci mismatching); ${ }^{\mathrm{d}}$ (Candidate Parent loci typed); ${ }^{\mathrm{e}}$ (Offspring-Candidate Parent loci mismatching); ${ }^{\mathrm{f}}(\mathrm{Offspring-Known} \mathrm{Par-}$ ent-Candidate Parent loci mismatching).

Exclusion power and null allele frequency estimates, for each locus and by enclosure, are presented in Table 2 . The overall probability of exclusion for the maternity test, by enclosure and considering one parent known $(\operatorname{Excl}(2))$, that is the case for this study, since the offsprings' father is always known as there was one single male by enclosure, was $99,1 \%$ for ARN1 (clutches 1 and 5), 96,4\% for ARN3 (clutches 2, 3 and 6) and 96,3\% for ARN4 (clutch 4).

According to the parentage test (Table 3 ) and on comparing genotypes (Table 4), the indicated mother for Clutch 1 is 4-CL106, in disagreement with the classification of female 2-CL25 as clutch-mother based solely on maternal behavior displayed by this individual and not the former. Nevertheless, the female 2-CL25 was excluded from maternity by six microsatellite markers, Ami 13 , Cla 02 ,

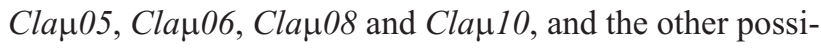
ble mother, 3-CL53, by five microsatellite markers,

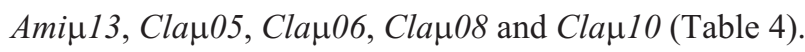

Female 34-CL10 was the behaviorally assigned mother of clutch 2 (Table 4). On the other hand, female 35-CL5 was assigned as the mother of 39-CL382 and 40-CL383 (Table 3), but she was excluded from maternity of the remaining hatchlings of clutch 2 by two microsatellite markers, $\mathrm{Cla \mu} 02$ and $\mathrm{Cla \mu} 09$ (Table 4). By the parentage test, female 36-CL13 was not assigned as mother, but could not be precluded from maternity of clutch 2 (Table 4). Female 37-CL14 was excluded from maternity of this clutch by microsatellite markers Ami 13 and Cla $\mu 09$, and female 38-CL70 was excluded from maternity of this

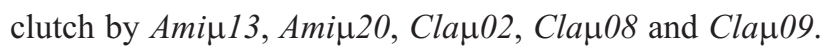
Maternity of clutch 2 remained uncertain for the females 34-CL10 and 36-CL13. Female 34-CL10 displayed parental behavior and was indicated as the mother by parentage testing of hatchlings 41-CL384, 42-CL385 and 43-CL386. In addition, she could not be definitely excluded as the mother of hatchlings 39-CL382 and 40-CL383. Female 


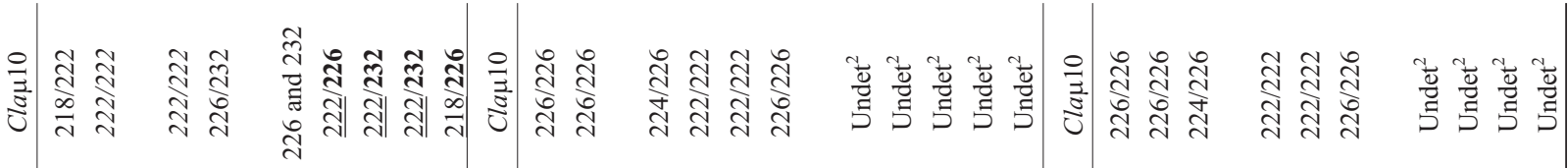

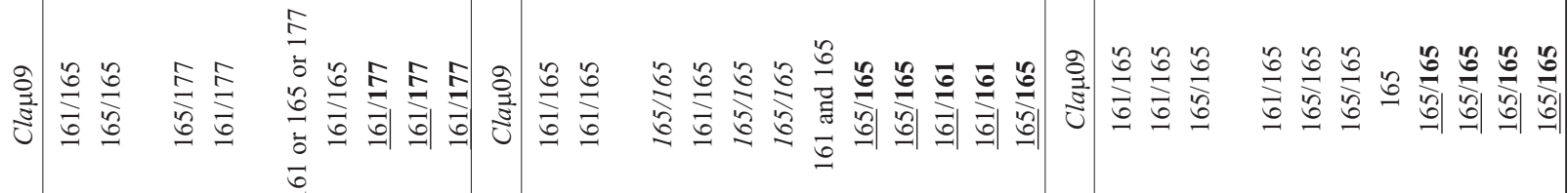

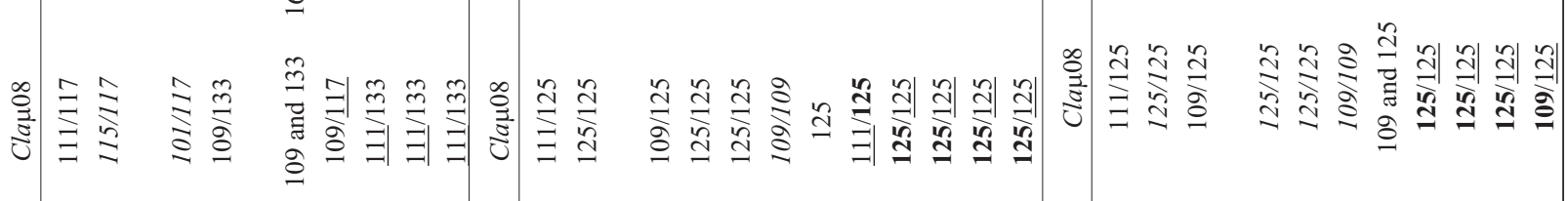

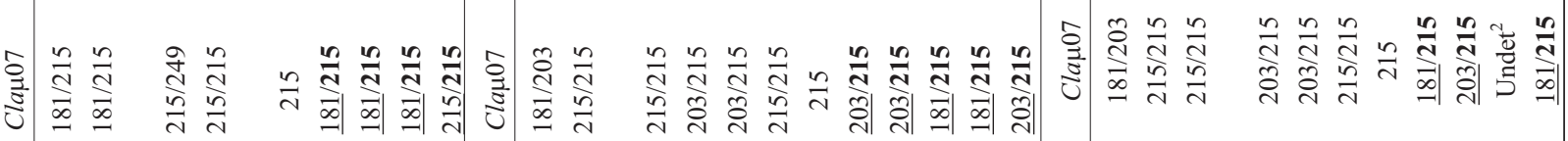

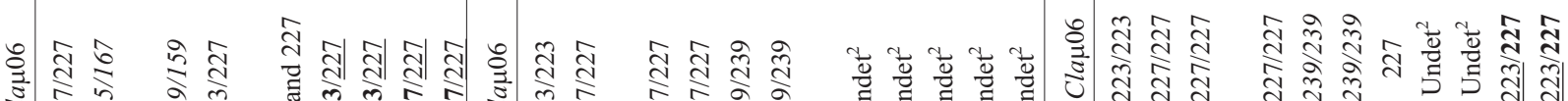

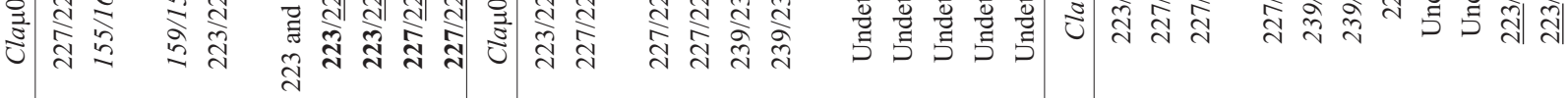

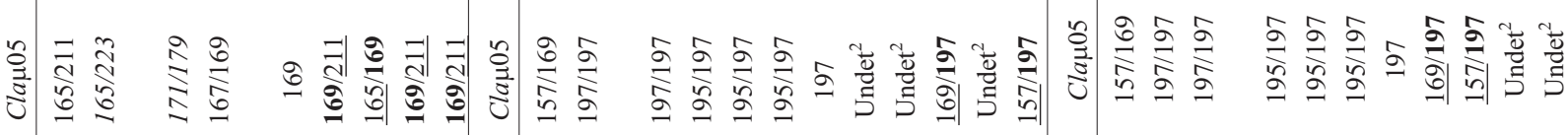

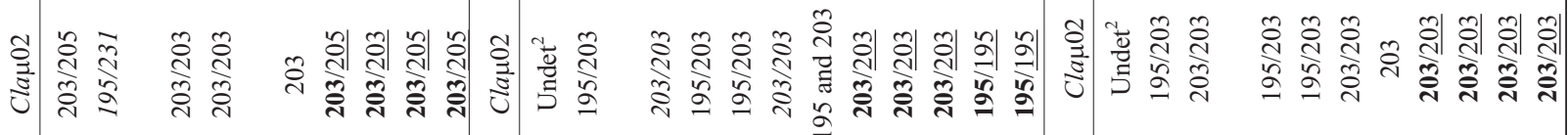

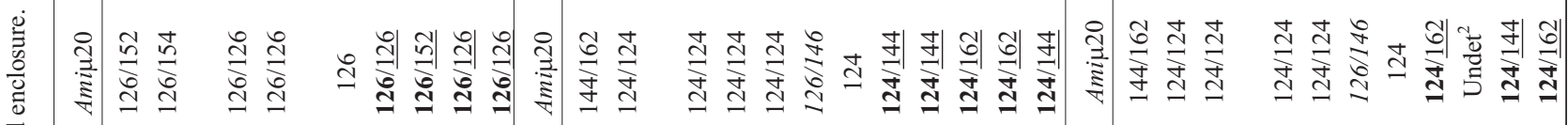

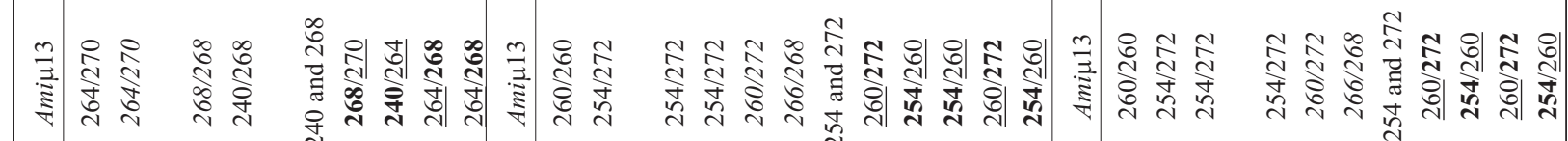

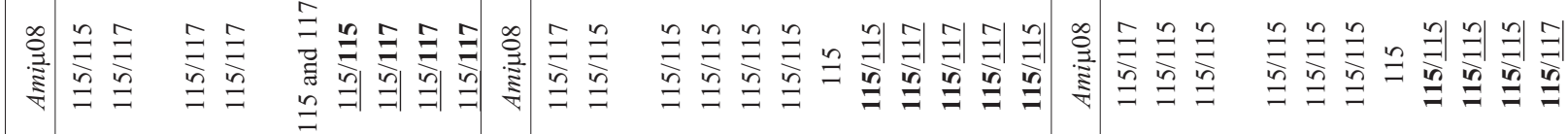




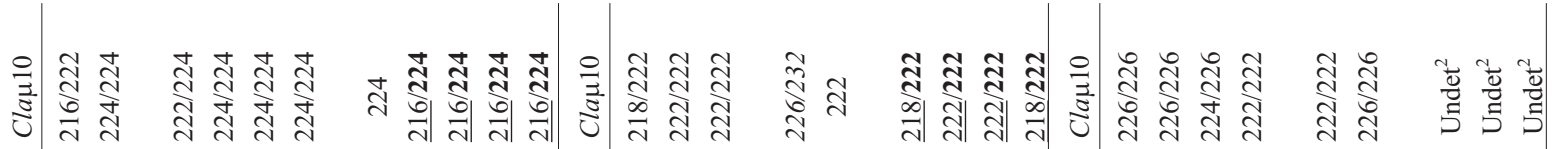

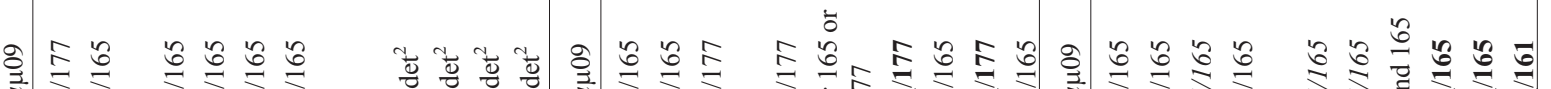

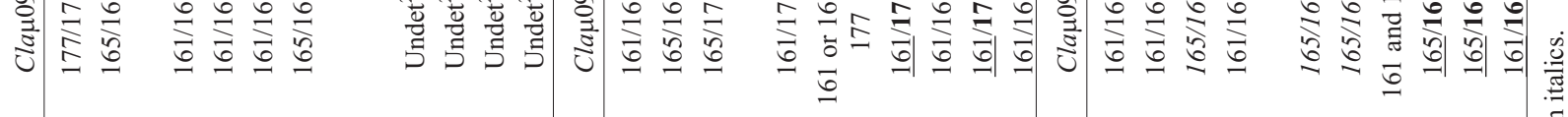

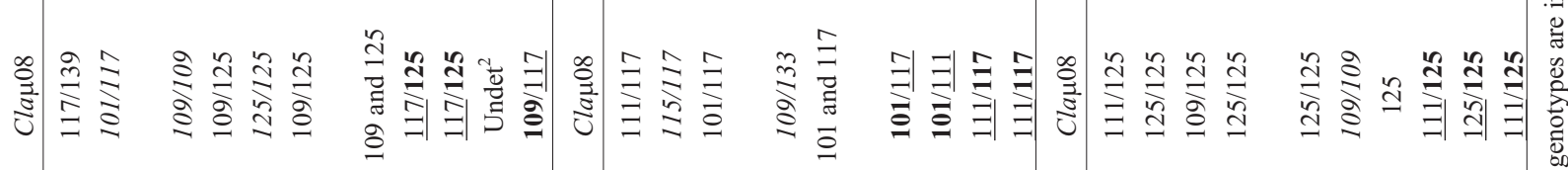

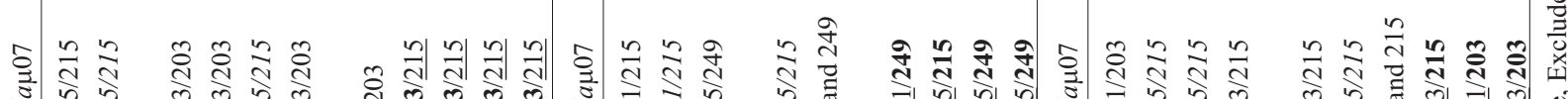

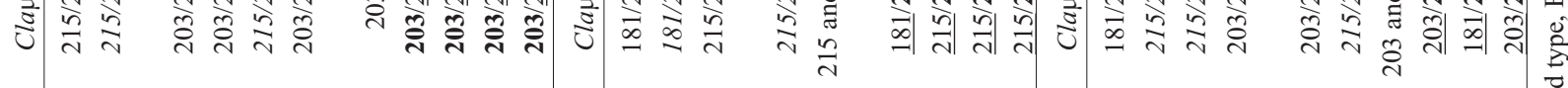

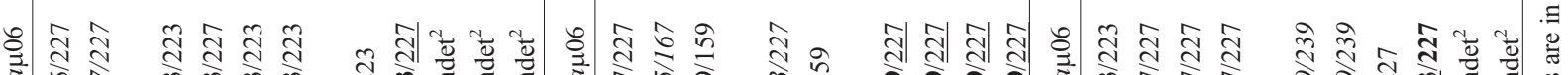

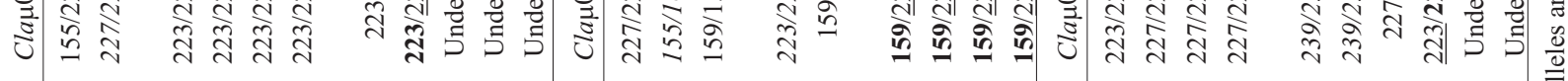

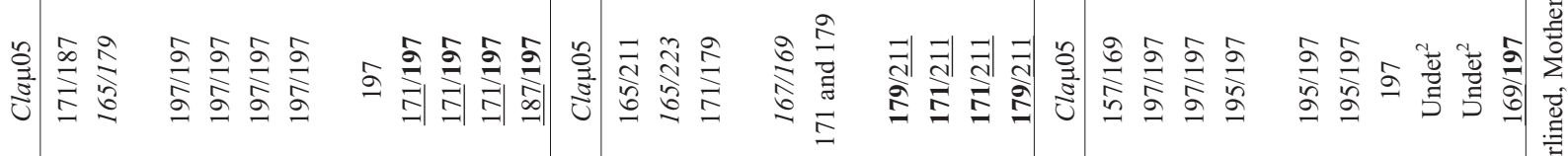

仓ิ

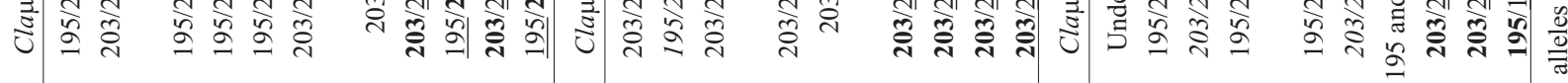

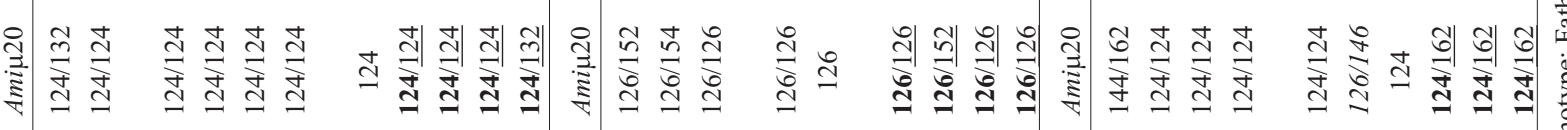

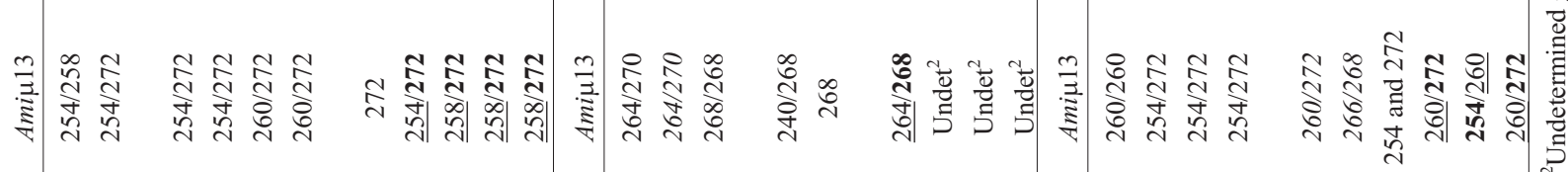

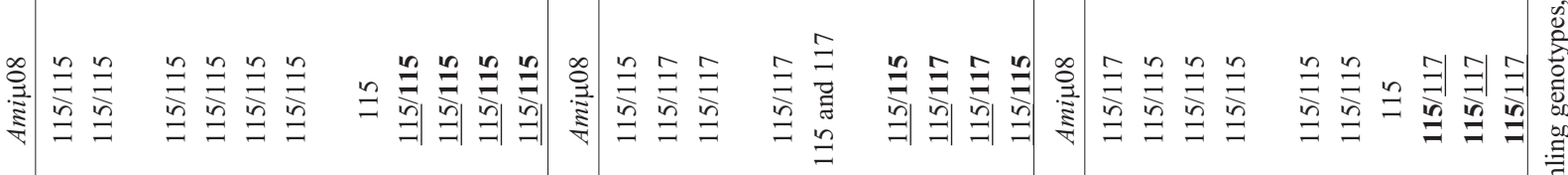

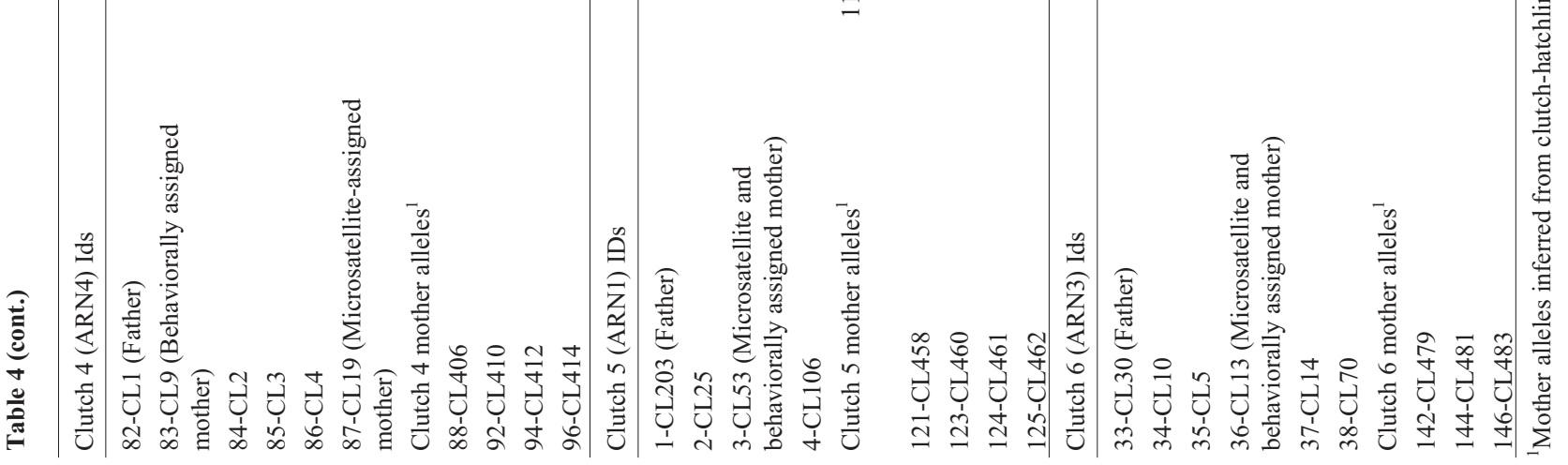


36-CL13 could not be excluded from maternity of this clutch by any microsatellite marker (Table 4), although she was not indicated as the mother of any of the hatchlings by the parentage test. This suggests that female 34-CL10 is the actual mother of clutch 2, based on both behavioral and microsatellite evidence.

Female 35-CL5 was assigned as the actual mother of clutch 3, based on both behavioral and microsatellite evidence (Tables 3 and 4). The remaining females in enclosure ARN3 were excluded from maternity of clutch 3 by several microsatellite markers (Table 4): female 34-CL10 was excluded by Cla 08 , female 36-CL13 by Cla 08 , female

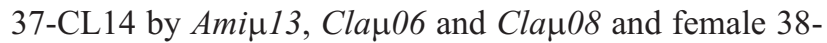

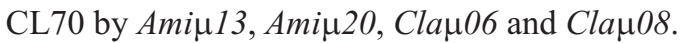

The behaviorally assigned mother of clutch 4,83 CL9, was excluded from maternity of this clutch by micro-

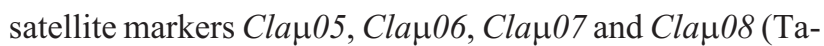
ble 4), whereas of the remaining females, 84-CL2 was excluded by $\mathrm{Cla \mu} 08$ and 86-CL4 by Cla 007 and $\mathrm{Cla \mu} 08$. Female 85-CL3 could be neither excluded from maternity, nor indicated as the mother through parentage testing. Female 87-CL19 could not be excluded from maternity (Table 4), but was assigned as mother through parentage testing (Table 3). This was another case in which the molecularly assigned mother (87-CL19) was different from the behaviorally assigned (83-CL9).

Female 3-CL53 was distinguished as the mother of clutch 5 by both parentage microsatellite analysis (Table 3 ) as well as maternal behavior. The other two females in the same enclosure (ARN1) were excluded as mothers by mi-

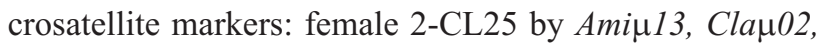

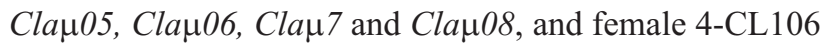

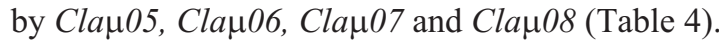

In clutch 6 , female 35 -CL5 was assigned as mother of 142-CL479 (Table 3), but was excluded from maternity of the remaining hatchlings by markers $\mathrm{Cla \mu} 02, \mathrm{Cla} \mu \mathrm{O}$ and Cla 09 (Table 4). Female 36-CL13 was indicated as mother of 144-CL481 and 146-CL483 (Table 3), and could not be excluded from the remaining hatchlings by comparison among genotypes (Table 4). Female 34-CL10 was excluded as mother by $\mathrm{Cla \mu} 07$, whereas female 37-CL14 was from maternity by $\mathrm{Ami \mu} 13, \mathrm{Cla \mu} 06$ and Cla $\mathrm{O} 09$ and female

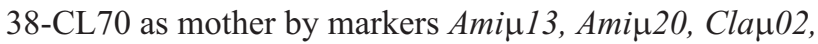

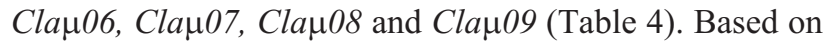
the above, female 36-CL13 was assigned as mother of the clutch through microsatellite analysis, which was also in accordance with behavioral displays.

In four of the six clutches $(2,3,5$ and 6$)$, mothers assigned by genetic analysis were in agreement with those indicated by maternal behavior: 34-CL10 for clutch 2, 35-CL5 for clutch 3 and 36-CL13 for clutch 6 (ARN3), 3-CL53 for clutch 5 (ARN1), see Tables 3 and 4.

For two of the six clutches ( 1 and 4$)$, mothers assigned by genetic analysis were not the same as those indi- cated by maternal behavior. Behaviorally assigned mother for Clutch 1 (ARN1) was 2-CL25, whereas 4-CL106 was indicated as mother by microsatellite assay parentage test (Tables 3 and 4). In Clutch 4 (ARN4), female 83-CL9 displayed maternal behavior, whereas female 87-CL19 was indicated as mother by microsatellite assay parentage test (Tables 3 and 4).

With the set of markers used, it was possible to identify a single mother for all the offspring: clutches 1 (4CL106), 2 (34-CL10), 3 (35-CL5), 4 (87-CL19), 5 (3CL53) and 6 (36-CL13). Surprisingly, two of the females $(33 \%)$ that displayed maternal behavior were not confirmed as actual mothers: 2-CL25 and 83-CL9. A display of maternal behavior by nonmothers can be explained as either a behavioral malfunction caused by the captive environment or species social adaptation as described in other vertebrates (Wrangham and Rubestein, 1986). Both hypotheses can be tested in future studies.

Farming operations are based on captive breeding and generally involve a small number of founders. Therefore, they require effective genetic management, in order to prevent genetic disorders as inbreeding depression (Foose, 1980). Assignment of mothers based exclusively on behavioral displays can lead to errors when assembling a Studbook and in establishing individual pedigrees. Under these circumstances microsatellite markers might be useful. In addition, these markers can also be useful in demographic and behavioral ecological studies in which the mating system and dispersal pattern are assessed based on parentage among individuals (e.g., Verdade et al., 2002).

\section{Acknowledgments}

We would like to thank Prof. Roland Vencovsky from the Departamento de Genética, ESALQ/USP, Piracicaba, São Paulo, Brazil, Dr. Travis C. Glenn of Savannah River Ecology Laboratory, PO Drawer E, Aiken, SC, USA and Dr. Luciana C. A. Regitano from the Centro de Pesquisa de Pecuária do Sudeste, EMBRAPA, São Carlos, São Paulo, Brazil for their help with molecular and statistical analyses. We also extend our thanks to Maria Antonia Ladalardo Echegaray, Nirlei Aparecida Silva, Lucia Elvira Alvares, Cláudia de Barros Monteiro-Vitorello, Mariângela Cristófani and Humberto Maciel França Madeira for their precious suggestions on molecular biology, as well as to Erica Elias Baron for her valuable suggestions on descriptive analysis. CNPq and FAPESP, respectively provided a masters and a doctorate scholarship to Rodrigo Barban Zucoloto.

\section{References}

Ballou JD (1992) Genetic and demographic considerations in endangered species captive breeding and reintroduction programs. In: McCulloughand DR and Barrett RH (eds) Wildlife Populations. Elsevier Applied Sciences, London, pp 262-275. 
Bernardes AT, Machado ABM and Rylands AB (1990) Fauna Brasileira Ameaçada de Extinção. IBAMA, Brasília, 62 pp.

Brazaitis P, Yamashita C and Rebelo G (1988) CITES Central South American Caiman Study Phase I: Central and Southern Brazil. Convention on International Trade of Endangered Species of Wild Fauna and Flora, Geneva, 62 pp.

Craighead L, Paetkau D, Reynolds HV, Vyse ER and Strobek C (1995) Microsatellite analysis of paternity and reproduction in arctic grizzly bears. J Hered 86:255-261.

Davis LM, Glenn TC, Dessauer HC, Elsey RM and Sawyer RH (2001) Multiple paternity in the American alligator, Alligator mississipiensis. Mol Ecol 10:1011-1024.

Foose TJ (1980) Demographic management of endangered species in captivity. Int Zoo Yearbook 20:154-66.

Garcia-Moreno J, Matocq MD, Roy MS, Geffen E and Wayne RK (1996) Relationships and genetic purity of endangered Mexican Wolf based on analysis of microsatellite loci. Conserv Biol 10:376-389.

Glenn TC, Dessauer HC and Braun MJ (1998) Characterization of microsatellite DNA loci in American alligators. Copeia 1998:591-601.

Glenn TC, Stephan W, Dessauer HC and Braun MJ (1996) Allelic diversity in Alligator microsatellite loci is negatively correlated with GC content of flanking sequences and evolutionary conservation of PCR amplifiability. Mol Biol Evol 13:1151-1154.

Groombridge B (1982) IUCN Amphibia - Reptilia Red Data Book Part 1. Testudines, Crocodylia, Rhyncocephalia. IUCN Conservation Monitoring Centre, Cambridge, 469 pp.

Hoelzel AR (1992) Molecular Genetic Analysis of Populations. IRL Press, Oxford, 315 pp.

Marshall TC, Slate J, Kruuk L and Pemberton JM (1998) Statistical confidence for likelihood-based paternity inference in natural populations. Mol Ecol 7:639-655.

Moore SS, Sargeant LL, King TJ, Mattick JS, Georges M and Hetzel JS (1991) The conservation of dinucleotide microsatellite among mammalian genomes allows the use of heterologous PCR primer pairs in closely related species. Genomics 10:654-660.

Olson GA, Hessler JR and Faith RE (1975) Techniques for blood collection and intravascular infusion of reptiles. Lab Anim Sci 25:783-786.

Palo J, Varvio S, Hanski I and Väinölä R (1995) Developing microsatellite markers for insect population structure, complex variation in checkerspot butterfly. Hereditas 123:295300.

Sambrook JE, Fritsch EF and Maniatis T (1989) Molecular Cloning: A Laboratory Manual. Cold Spring Harbor Laboratory Press, New York.

Thorbjarnarson JB (1996) Reproductive characteristics of the order Crocodylia. Herpetologica 52:8-24.

Vanzolini PE (1972) Répteis e anfíbios ameaçados de extinção no Brasil. In: Espécies da Fauna Brasileira Ameaçadas de Extinção. Academia Brasileira de Ciências, Rio de Janeiro, pp 155-157.

Verdade LM (1995) Biologia reprodutiva do jacaré-de-papo-amarelo (Caiman latirostris) em São Paulo, Brasil. In: Larriera A and Verdade LM (eds) Conservación y Manejo de los
Crocodylia de America Latina, v. 1. Fundación Banco Bica, Santo Tomé, pp 57-79.

Verdade LM (1997). Manejo e conservação do jacaré-de-papo-amarelo (Caiman latirostris) no Estado de São Paulo. In: Valladares-Padua C, Bodmer RE and Cullen Jr L (eds) Manejo e Conservação de Vida Silvestre no Brasil. CNPq, Brasília, pp 222-232.

Verdade LM (1998) Caiman latirostris. In: Ross JP (ed) Crocodile Status Survey an Conservation Action Plain. The World Conservation Union, Gland, pp 18-20.

Verdade LM (2001) O programa experimental de criação em cativeiro do jacaré-de-papo-amarelo (Caiman latirostris) da ESALQ / USP: Histórico e perspectivas. In: Mattos WRS (ed) A Produção Animal na Visão dos Brasileiros. Sociedade Brasileira de Zootecnia, Piracicaba, pp 559-564.

Verdade LM and Andrade RN (2003) Studbook Regional do Jacaré-de-Papo-Amarelo (Caiman latirostris): 1993/2003. ESALQ / USP, Piracicaba, São Paulo.

Verdade LM and Kassouf-Perina S (1993) Studbook Regional do Jacaré-de-Papo-Amarelo (Caiman latirostris): 1992/1993. Sociedade de Zoológicos do Brasil, São Paulo.

Verdade LM and Piña CI (2006). Caiman latirostris. Catalog of the American Society of Amphibians and Reptiles 833:1-21.

Verdade LM and Sarkis F (1998) Age at first reprodution in captive Caiman latirostris (Broad-snouted Caiman). Herpetol Rev 29:227-228.

Verdade LM, Michelotti F, Rangel MC, Cullen Jr L, Ernandes MM and Lavorenti A (1992) Manejo dos ovos de jacarésde-papo-amarelo (Caiman latirostris) no CIZBAS / ESALQ / USP. In: Verdade LM and Lavorenti A (eds) Anais do II Workshop sobre Conservação e Manejo do Jacaré-de-Papo-Amarelo (Caiman latirostris). CIZBAS / ESALQ / USP, Piracicaba, pp 92-99.

Verdade LM, Zucoloto RB and Coutinho LL (2002) Microgeographic variation in Caiman latirostris. J Exp Zool 294:387-396.

Verdade LM, Sarkis-Gonçalves F, Miranda-Vilella MP and Bassetti LAB (2003) New record of age at sexual maturity in captivity for Caiman latirostris (broad-snouted caiman). Herpetol Rev 34:225-226.

Wrangham RW and Rubestein DI (1986) Social evolution in birds and mammals. In: Rubestein DI and Wrangham RW (eds) Ecological Aspects of Social Evolution: Birds and Mammals. Princeton University Press, Princeton, pp 452-470.

Zucoloto RB, Verdade LM and Coutinho LL (2002) Microsatellite DNA library for Caiman latirostris. J Exp Zool 294:346.

\section{Internet Resources}

IBAMA (2003) Lista das Espécies Brasileiras Ameaçadas de Extinção. Anexo à Instrução Normativa No. 3 de 27 de maio de 2003 do Ministério do Meio Ambiente http://www.ibama.gov.br/fauna/downloads/lista\%20spp.pdf (October 10, 2008).

Associate Editor: João S. Morgante

License information: This is an open-access article distributed under the terms of the Creative Commons Attribution License, which permits unrestricted use, distribution, and reproduction in any medium, provided the original work is properly cited. 